\title{
Biomasse, productivité et transferts de matière organique dans une séquence altitudinale de peuplements de Castanea sativa Mill de l'Etna
}

\author{
S Leonardi ${ }^{1,2}$, M Rapp $^{3}$, M Failla ${ }^{1}$, D Guarnaccia ${ }^{1}$ \\ 1 Facoltà di Scienze Matematiche, Fisiche e Naturali, Università di Catania, \\ 19 Via A Longo, 95125 Catania ; \\ ${ }^{2}$ Corso di Laurea in Scienze Forestali ed Ambientali, Università di Palermo, \\ 23 Via Roma, 92010 Bivona, Italie; \\ ${ }^{3}$ CNRS-CEFE, BP 5051, 34033 Montpellier cedex, France
}

(Reçu le 3 octobre 1994 ; accepté le 19 septembre 1995)

\begin{abstract}
Summary - Biomass, productivity and organic matter fluxes in Castanea sativa Mill stands of various elevation in the Etna volcano. All the compartments and fluxes of organic matter distribution and turnover within four Castanea sativa Mill coppice stands of various ages and at various elevations were studied on the flanks of the Etna volcano (Sicily). Aboveground biomass was to 22 and $24 \mathrm{Mg}$ $\mathrm{ha}^{-1}$ in 7-year-old stands and 83 and $100 \mathrm{Mg} \mathrm{ha}^{-1}$ in 12- and 22-year-old coppices, with good correlations between biomass and climatic conditions or stand age. Wood accounted for $95 \%$ of the biomass and leaves for $5 \%$. Aboveground perennial biomass production amounted to 2.4 to $5.4 \mathrm{Mg} \mathrm{ha}^{-1}$ year ${ }^{-1}$, and thus was also correlated with the age and the altitudinal location of the stands. Litterfall, measured during 3 successive years, varied between 1.7 and $5.1 \mathrm{Mg} \mathrm{ha}^{-1}$ year ${ }^{-1}$ and was directely related to the stand age. The litter accumulation on the soil surface was 7.4 to $7.9 \mathrm{Mg} \mathrm{ha}^{-1}$, with only small differences between the four sites. Decomposition required more then 6 years. The comparison of the four coppices showed that all the processes concerning productivity, as well as decomposition, were related to climatic conditions and to the age of the coppice stands.
\end{abstract}

Castanea sativa Mill / organic matter / biomass / productivity / litterfall / litter decomposition

Résumé - Les différents compartiments et flux caractérisant la répartition de la matière organique sont étudiés dans quatre taillis de Castanea sativa Mill, étagés le long d'un gradient altitudinal, dans le massif de l'Etna (Sicile). Les biomasses épigées sont respectivement de 22 et de $24 \mathrm{Mg} \mathrm{ha}^{-1}$ à 7 ans, $83 \mathrm{Mg} \mathrm{ha}^{-1}$ à 12 ans et $100 \mathrm{Mg} \mathrm{ha}^{-1}$ à 22 ans, avec de bonnes corrélations : biomasse/âge et biomasse/facteurs climatiques. Le bois représente $95 \%$ et les feuilles $5 \%$ de ces biomasses. Les productions aériennes pérennes s'échelonnent entre 2,4 et $5,4 \mathrm{Mg} \mathrm{ha}^{-1} \mathrm{an}^{-1}$ en relation à la fois avec l'âge des taillis et leur localisation géographique. Les retombées de litière, observées durant 3 années consécutives, se situent entre 1,7 et $5,1 \mathrm{Mg} \mathrm{ha}^{-1} \mathrm{an}^{-1}$. Elles sont directement fonction de l'âge des peuplements. Cette litière reste immobilisée à la surface du sol, où elle représente une masse de 7,4 à $7,9 \mathrm{Mg} \mathrm{ha}^{-1}$, donc peu variable d'une station à l'autre. La décomposition de cette litière s'étend sur une période supérieure à 6 années. La comparaison des quatre taillis étudiés indique que l'ensemble des processus impliqués, aussi bien dans la production que dans la décomposition de la matière 
organique, est fonction d'une part des facteurs climatiques stationnels ou globaux, d'autre part de l'âge des peuplements.

Castanea sativa Mill / matière organique / biomasse / litière / décomposition / production

\section{INTRODUCTION}

Dans le domaine forestier, les peuplements de châtaigniers (Castanea sativa Mill) occupent en Italie le second rang en ce qui concerne la surface, après les différents peuplements de chênes, et ce tout au long du pays, depuis les Alpes jusqu'en Sicile. Au niveau économique, cette essence, méditerranéenne et calcifuge, occupe également la seconde place pour la production de bois et constitue une source importante de revenus par sa production fruitière (Boggia, 1988).

En Sicile (Asciuto et al, 1988), les forêts de Castanea sativa ne couvrent qu'une surface réduite, de l'ordre de 4000 ha, soit moins de $1 \%$ de la surface occupée sur l'ensemble de l'Italie, dont plus de la moitié dans le massif de l'Etna. Castanea sativa y est implanté depuis fort longtemps (Scuderi, 1825), plus particulièrement sur les versants sud et est du volcan, entre 600 et $1600 \mathrm{~m}$ d'altitude.

L'existence de peuplements de différents âges, localisés à différentes altitudes, mais sur un substrat géologique identique et des sols voisins, constitue dans le massif de l'Etna un bon modèle, à la fois synchronique et diachronique, pour l'étude du fonctionnement des écosystèmes à Castanea sativa.

La présente étude porte sur la production de biomasse épigée, la répartition et les flux de matière organique dans quatre peuplements du parc de l'Etna. Deux peuplements sont implantés à basse altitude (1 000$1200 \mathrm{~m}$ ), sur le versant oriental du volcan, deux autres entre 1400 et $1600 \mathrm{~m}$ d'altitude avec exposition sud. Chaque couple est constitué lui-même d'un taillis jeune, âgé de 7 ans au début de l'étude, et d'un taillis âgé arrivé plus ou moins à maturité.
Ce travail débouche à la fois sur la connaissance du fonctionnement des systèmes à Castanea sativa au niveau de la production et de la répartition de la biomasse aérienne, en relation avec les facteurs climatiques ou avec l'âge des peuplements et sur des indications permettant l'amélioration de leur gestion sylvicole, en relation avec l'environnement.

\section{DESCRIPTION DES STATIONS}

\section{Localisation}

Les quatre peuplements ont été sélectionnés le long d'un gradient altitudinal. Les deux sites d'altitude sont localisés sur la face sud de l'Etna, entre 1400 et $1600 \mathrm{~m}$ d'altitude, sur une pente de $30 \%$ pour la station de Fossa la Nave, de $10 \%$ pour celle de Balilla (fig 1).

La station de Monte Crisimo, implantée sur d'anciennes terrasses, et celle de Piano Porcheria se situent sur le versant oriental du volcan et sont représentatives des châtaigneraies de basse altitude, reconnue les plus productives.

\section{Sol}

Les quatre stations se trouvent sur des laves et des cendres volcaniques basaltiques, qui selon les dates des dernières coulées sont âgées de 2000 à 2000000 d'années. Les sols des quatre stations sont très proches, de type andosol humifié, de 50 à $60 \mathrm{~cm}$ d'épaisseur en moyenne. Ces sols se subdivisent en un horizon $A_{1}$, riche en matière organique, de 1 à $15 \mathrm{~cm}$ d'épaisseur, suivi d'un horizon $\left(A_{1} B\right)$ de 15 $60 \mathrm{~cm}$, moins organique, très riche en cendres ou en laves dégradées, bien colonisés par les racines. 


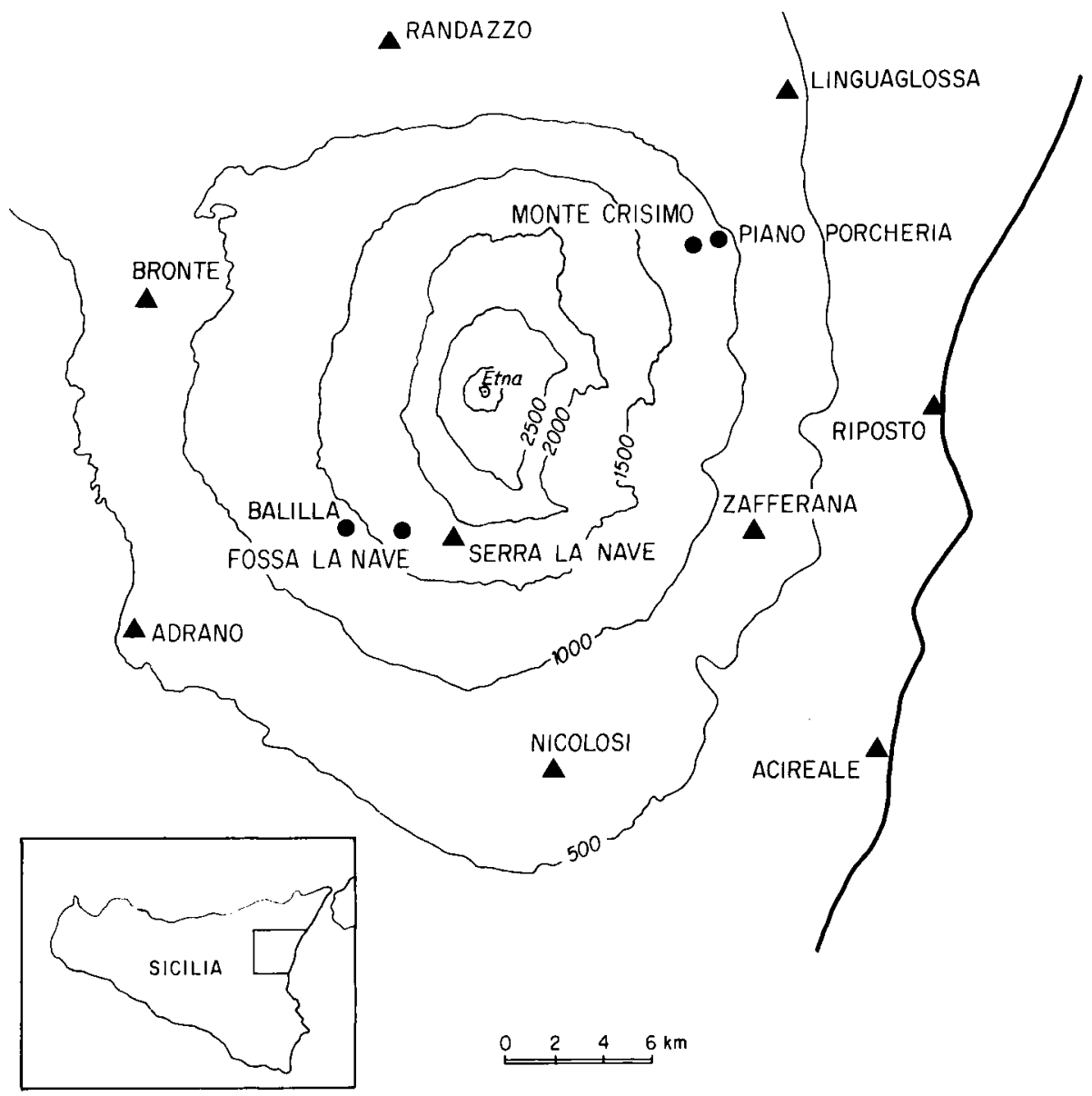

Fig 1. Localisation des quatre stations de Castanea sativa Mill dans le massif de l'Etna (cercles pleins).

En dessous de $60-70 \mathrm{~cm}$ on ne rencontre que des sables, scories ou laves volcaniques en blocs.

Les caractéristiques chimiques des sols des quatre stations sont résumées sur le tableau I.

\section{Climat}

Le climat est de type mésoméditerranéen xérothermique pour les stations de faible altitude. La station de Fossa la Nave et à un moindre degré celle de Balilla sont caractéristiques d'un climat de montagne, à hiver froid, avec régulièrement de la neige et une pluviométrie moindre. Dans tous les peuplements, la période de sécheresse estivale est de 3 mois. La figure 2 résume les variations des températures et des précipitations moyennes mensuelles de stations météorologiques situées au voisinage des parcelles étudiées. 
Tableau I. Propriétés chimiques des sols des quatre stations étudiées.

\begin{tabular}{|c|c|c|c|c|c|c|c|c|}
\hline \multirow{2}{*}{$\begin{array}{r}\text { Stations: } \\
\text { Profondeur sol } \mathrm{cm} \text { : }\end{array}$} & \multicolumn{2}{|c|}{ Fossa la Nave } & \multicolumn{2}{|c|}{ Balilla } & \multicolumn{2}{|c|}{ Piano Porcheria } & \multicolumn{2}{|c|}{ Monte Crisimo } \\
\hline & 20 & 50 & 20 & 50 & 20 & 50 & 20 & 50 \\
\hline$p H:$ & 6,7 & 6,7 & 6,4 & 6,5 & 6,6 & 6,6 & 6,5 & 6,5 \\
\hline$C \%$ & 2,52 & 2,18 & 2,85 & 2,27 & 1,94 & 1,90 & 2,14 & 1,94 \\
\hline $\mathrm{N} \%$ & 0,18 & 0,18 & 0,19 & 0,16 & 0,15 & 0,17 & 0,19 & 0,16 \\
\hline $\mathrm{C} / \mathrm{N}$ & 14 & 13 & 15 & 14 & 13 & 11 & 11 & 13 \\
\hline M Org, \% & 4,33 & 3,75 & 4,90 & 3,90 & 3,34 & 3,27 & 3,68 & 3,34 \\
\hline CEC méq \% sol & 13,7 & 15,2 & 13,3 & 12,2 & 9,2 & 10,1 & 10,7 & 11,2 \\
\hline $\mathrm{Ca}$ & 7,50 & 8,00 & 7,50 & 6,90 & 4,90 & 4,60 & 4,60 & 4,30 \\
\hline $\mathrm{Na}$ & 0,09 & 0,11 & 0,09 & 0,09 & 0,06 & 0,06 & 0,08 & 0,06 \\
\hline $\mathrm{Mg}$ & 1,82 & 1,79 & 1,78 & 1,46 & 1,14 & 1,24 & 1,38 & 1,19 \\
\hline $\mathrm{K}$ & 0,59 & 0,56 & 0,51 & 0,51 & 0,28 & 0,32 & 0,35 & 0,30 \\
\hline$S / T \%$ & 73 & 69 & 74 & 73 & 61 & 62 & 60 & 52 \\
\hline \multicolumn{9}{|l|}{$\begin{array}{l}\text { Éléments totaux } \\
\%\end{array}$} \\
\hline $\mathrm{Ca}$ & 5,64 & 5,53 & 5,62 & 5,66 & 5,77 & 5,48 & 5,25 & 4,98 \\
\hline $\mathrm{Mg}$ & 2,03 & 1,89 & 2,01 & 2,00 & 2,02 & 1,95 & 1,91 & 1,70 \\
\hline$K$ & 1,20 & 1,11 & 1,21 & 1,23 & 1,25 & 1,24 & 1,23 & 1,16 \\
\hline $\mathrm{Na}$ & 2,44 & 2,42 & 2,46 & 2,51 & 2,64 & 2,56 & 2,57 & 2,48 \\
\hline $\mathrm{P}$ & 0,64 & 0,64 & 0,62 & 0,63 & 0,61 & 0,61 & 0,61 & 0,66 \\
\hline$S$ & 0,04 & 0,04 & 0,07 & 0,04 & 0,07 & 0,10 & 0,07 & 0,06 \\
\hline
\end{tabular}

\section{Végétation}

Les châtaigneraies de l'Etna, toutes introduites depuis fort longtemps, occupent l'aire naturelle des chênes caducifoliés mesoméditerranéens (Ronsisvalle et Signo- rello, 1979 ; Poli et al, 1981). Ceci explique l'absence d'une association caractéristique à ces groupements.

Les peuplements sont constitués d'une strate arborée, formée essentiellement de rejets sur souche de Castanea sativa. Le

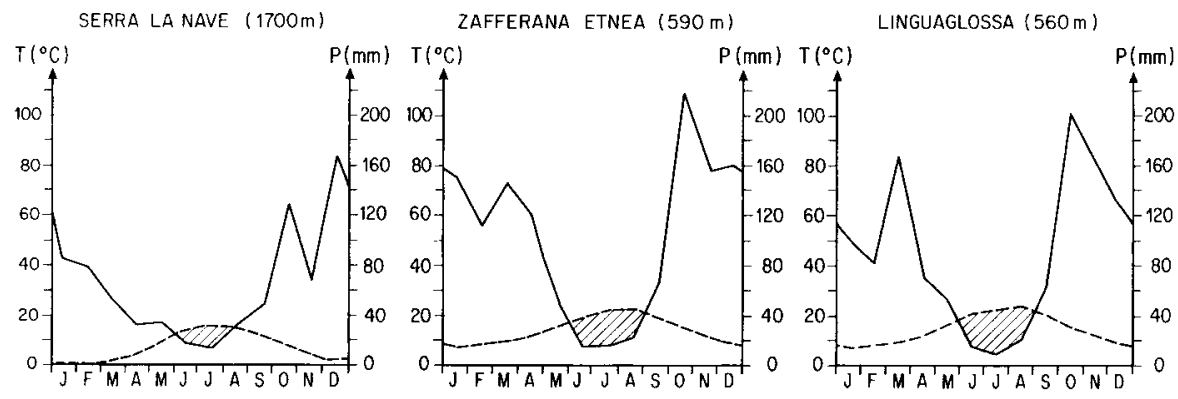

Fig 2. Diagrammes ombrothermiques de trois stations météorologiques situées à proximité des peuplements étudiés : Serra la Nave pour Fossa la Nave et Balilla ; Zafferana Etnea et Linguaglossa pour Monte Crisimo et Piano Porcheria. 
Tableau II. Caractéristiques des quatre stations de Castanea sativa Mill de l'Etna.

\begin{tabular}{|c|c|c|c|c|c|c|c|c|}
\hline Station & $\begin{array}{l}\text { Altitude } \\
(m)\end{array}$ & $\begin{array}{c}\text { Cépées } \\
\left(h a^{-1}\right)\end{array}$ & $\begin{array}{c}\text { Rejets } \\
\left(t_{0^{*}}\right)\end{array}$ & $\begin{array}{c}\text { Rejets } \\
\left(t+2 / 3^{\star *}\right)\end{array}$ & $\begin{array}{l}\text { Rejets } \\
\text { par } \\
\text { cépée } \\
\text { (to) }\end{array}$ & $\begin{array}{c}\text { Rejets } \\
\text { par } \\
\text { cépée } \\
(t+2 / 3)\end{array}$ & $\begin{array}{c}\text { Surface } \\
\text { terrière } \\
\left(m^{2} h a^{-1}\right)\end{array}$ & $\begin{array}{c}\text { Diamètre } \\
\text { moyen } \\
\text { du tronc } \\
\text { à } 1,30 \mathrm{~m} \\
(\mathrm{~cm})\end{array}$ \\
\hline Fossa la Nave & 1600 & 238 & 1895 & 1876 & 8 & 7 & 8,4 & 7,5 \\
\hline Balilla & 1400 & 245 & 1529 & 1376 & 6 & 6 & 30,9 & 16,0 \\
\hline Monte Crisimo & 1200 & 441 & 5529 & 5199 & 12 & 12 & 29,1 & 8,2 \\
\hline Piano Porcheria & 1000 & 288 & 5668 & 4955 & 20 & 17 & 10,5 & 4,9 \\
\hline
\end{tabular}

${ }^{*} t_{0}=$ année initiale. ${ }^{* \star} t+2 / 3=$ après 2 années (après 3 années : Fossa la Nave).

tableau II et la figure 3 résument la répartition des tiges de Castanea sativa en cépées et rejets ainsi qu'en classes de diamètre.

La strate herbacée très réduite, se limite essentiellement à Pteridium aquilinum.

\section{METHODES D'ÉTUDE}

\section{Biomasse}

La biomasse a été déterminée en établissant des relations mathématiques entre un paramètre dendrométrique facile à mesurer et les poids secs d'un certain nombre d'arbres abattus ou de leurs organes respectifs. Cette approche est préconisée et utilisée par de nombreux auteurs et a été décrite en détail par Bouchon et al (1985) et plus récemment par Hanchi (1994).

Pour l'ensemble des quatre stations, 36 rejets, couvrant les différentes classes de diamètre, entre 0,5 et $35,3 \mathrm{~cm}$, ont été coupés, puis séparés en tronc et en branches ; 22 individus ont été abattus en hiver, 14 durant la période de végétation, pour établir la biomasse de feuilles.

Pour tous les individus on a séparé les branches du tronc et les feuilles des branches. Les différentes fractions ont été pesées sur place, des sous-échantillons représentatifs (rondelles du tronc à différentes hauteurs, branches de différents diamètres et longueurs) ont été trans- férés au laboratoire, repesés puis séchés à l'étuve à $85^{\circ} \mathrm{C}$ jusqu'à poids constant. Cela permet de calculer la teneur en eau des arbres avec un maximum de précision.

L'ensemble de ces sous-échantillons constitue également le substrat analytique pour une étude du cycle biogéochimique dans ces mêmes peuplements.

Après détermination de l'âge des arbres abattus et établissement du poids sec des troncs, des branches et des feuilles des 36 arbres étudiés, on a calculé des équations de régressions entre le diamètre à $1,30 \mathrm{~m}$ du tronc et le poids fractionné ou total des arbres. Ce sont les régressions de puissance qui donnent les meilleurs résultats (Baskerville, 1965). Elles sont de type:

$$
Y=\mathbf{a} \times X^{\circ}
$$

avec $Y$ : poids de tronc, de branches ou de feuilles ; $X$, diamètre en $\mathrm{cm}$ du tronc à $1,30 \mathrm{~m}$; a et $b$, constantes.

Ces équations, appliquées aux différentes classes de diamètre des individus de chaque station et multipliées par le nombre d'individus par classe, permettent d'établir la biomasse du peuplement

\section{Productivité}

Deux approches ont été utilisées. La première est basée sur la notion de productivité moyenne d'un écosystème depuis son origine, telle qu'elle a été définie par 

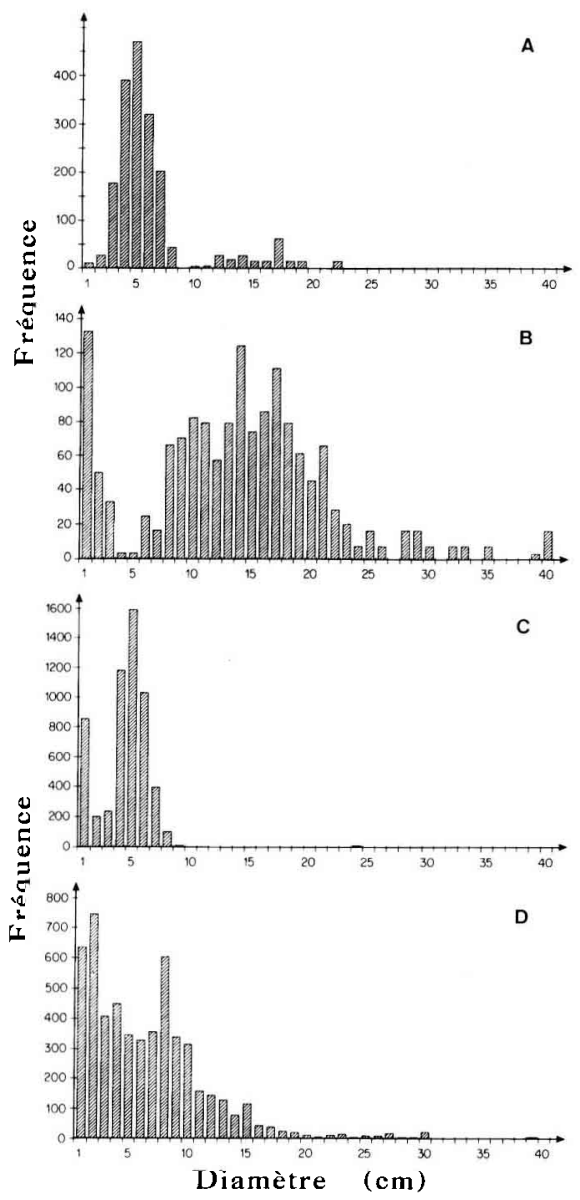

Fig 3. Distribution des rejets en classes de diamètre à $1,30 \mathrm{~m}$ dans les quatre stations étudiées: (A) Fossa la Nave, (B) Balilla, (C) Piano Porcheria et (D) Monte Crisimo.

Duvigneaud (1974) et Lemee (1974) : augmentation de la masse de matière vivante élaborée au cours d'un temps donné. Elle peut concerner un arbre pris individuellement ou un peuplement à un âge donné et sur une superficie déterminée. Dans ce travail, vu le nombre d'arbres étudiés, nous avons privilégié l'approche par arbre, beaucoup plus précise, quant à la détermi- nation exacte de l'âge, qu'un calcul global au niveau des quatre peuplements. On peut ainsi établir, à partir de la biomasse et de l'âge des arbres traités, toute une série de productions moyennes en fonction de leur âge.

Une seconde approche a consisté à déterminer la biomasse des peuplements par mensuration des circonférences de tous les individus des quatre peuplements à 2 ans (Balilla, Piano Porcheria, Monte Crisimo) ou 3 ans (Fossa la Nave) d'intervalle. La différence entre les valeurs des deux biomasses pérennes successives représente la production de chaque peuplement durant l'intervalle de temps donné.

Si cette approche ne pose pas de problème pour les peuplements les plus âgés de Balilla et de Monte Crisimo, le cas est différent pour les formations plus jeunes, la morphologie des rejets juvéniles pouvant varier avec l'âge. Mais après 6 ou 7 ans, les rejets des sites étudiés ont des tiges à morphologie d'adulte. Par ailleurs l'échantillon de 36 arbres utilisés pour calculer les biomasses inclut 17 individus de diamètre à $1,30 \mathrm{~m}$ inférieur à $10 \mathrm{~cm}$. La même approche peut donc être utilisée pour l'ensemble des quatre stations.

Pour la station de Piano Porcheria, on note également une très forte mortalité : 713 tiges sur un total de 5668 , soit $12 \%$ des individus. Ce même fait, déjà constaté par Ford et Newbould (1970), Ranger et al (1990b) et Bedeneau (1994), a nécessité une correction des estimations directes. Pour cela on a utilisé la première approche et calculé, sur un certain nombre de pieds bien identifiés, l'incrément de biomasse durant la période allant du 25 mars 1990 au 27 mars 1992. Celui-ci était en moyenne de $15,9 \%$ par an. Ce taux, appliqué à l'ensemble des tiges initialement présentes, permet d'établir une biomasse théorique, hors mortalité, de $31229 \mathrm{~kg} \mathrm{ha}^{-1}$, correspondant à une production moyenne annuelle optimale de $4381 \mathrm{~kg} \mathrm{ha}^{-1}$ de biomasse pérenne épigée, que l'on peut 
subdiviser elle même en $82 \%$ de bois de troncs et $18 \%$ de bois de branches.

\section{Surface foliaire}

Pour l'ensemble des rameaux feuillés transportés au laboratoire, on a déterminé le nombre total de feuilles puis établi leurs surfaces foliaires à l'aide d'un photoplanimètre automatique. Cela permet d'établir une relation entre le poids des feuilles et leur surface et de calculer l'indice foliaire (LAI $\mathrm{m}^{2} / \mathrm{m}^{2}$ ) au niveau de chaque station.

\section{Retombée de litière}

Dix collecteurs, d'un quart de mètre carré de surface de captage chacun, ont été disposés au hasard dans chacune des quatre stations. La litière a été récoltée mensuellement, ou à un rythme plus soutenu durant la phase automnale.

La litière récoltée est subdivisée en feuilles, bois, inflorescences, fruits et matériel divers non identifiable. Après séchage à $85^{\circ} \mathrm{C}$, les différentes fractions sont pesées puis leurs poids respectifs extrapolés à une superficie d'un hectare.

\section{Litière au sol}

Dix échantillons de litière au sol de $2500 \mathrm{~cm}^{2}$ de surface chacun (un quart de mètre carré) ont été prélevés dans chacune des quatre stations. Pour chaque échantillon, on a séparé le matériel foliaire des organes reproducteurs, essentiellement bogues et châtaignes et du matériel ligneux.

Pour éliminer toute contamination, soit directement à partir du sol, soit depuis d'éventuelles retombées solides, très fréquentes en milieu volcanique actif, on a calciné un certain nombre d'échantillons à $550{ }^{\circ} \mathrm{C}$ et exprimé les résultats en matière organique.

\section{Décomposition de la litière}

Dans chaque peuplement, des échantillons de $10 \mathrm{~g}$ de feuilles séchées ont été placés dans des filets en nylon de $2 \mathrm{~mm}$ de maille (litter-bags), disposés au milieu de la litière en place. Trois sacs par station sont récupérés tous les 2 mois durant 2 années consécutives, séchés et pesés.

Cette approche, initialement décrite par Burges (1956) et Bocock et Gilbert (1957), a été depuis utilisée par de nombreux chercheurs.

\section{RÉSULTATS ET DISCUSSION}

\section{Biomasse et production épigée}

À partir des 36 arbres abattus et débités, on a établi les équations de régression suivantes, permettant de calculer les différentes fractions de la biomasse d'un arbre $(Y$ ) en gramme en fonction du diamètre du tronc à $1,30 \mathrm{~m}(X)$ en centimètre :

$$
\begin{array}{lll}
Y_{\text {bois }}=96,771 \times X^{2,318} & n=36 & r=0,981 \\
Y_{\text {feuilles }}=3,023 \times X^{2,414} & n=14 & r=0,967 \\
Y_{\text {biomasse }}=102,000 \times X^{2,314} & n=36 & r=0,982
\end{array}
$$

Appliquées aux populations des quatre stations étudiées, ces équations permettent d'établir les biomasses épigées respectives suivantes : 22,0 et $24,1 \mathrm{Mg} \mathrm{ha}^{-1} 7$ ans après la dernière coupe à Fossa la Nave et à Piano Porcheria, 83,2 $\mathrm{Mg} \mathrm{ha}^{-1} 12$ ans après la dernière coupe à Monte Crisimo et $99,6 \mathrm{Mg} \mathrm{ha}^{-1}, 22$ ans après coupe à Balilla (tableau III).

Ces valeurs sont inférieures aux $107 \mathrm{Mg}$ ha $^{-1}$ de biomasse citée par La Marca (1984) pour un peuplement de Castanea sativa d'Italie âgé de 13 ans et proches des évaluations de Berthier (1984) en France. Ce dernier auteur détermine des biomasses aériennes pérennes (tronc et branches) de $25,2,37,8$ et $67,8 \mathrm{Mg} \mathrm{ha}^{-1}$ à 5,8 et 11 ans respectivement. Ranger et al (1990b) indiquent des valeurs voisines avec 
Tableau III. Biomasse des stations en $\mathrm{kg} \mathrm{ha}^{-1}$.

\begin{tabular}{|c|c|c|c|c|c|}
\hline & \multicolumn{4}{|c|}{ Biomasses fractionnées } & \multirow{2}{*}{$\begin{array}{c}\text { Biomasse } \\
\text { totale }\end{array}$} \\
\hline & Tronc & Branches & Bois & Feuilles & \\
\hline Fossa la Nave & 16688,7 & 3741,3 & 20430,0 & 1607,2 & 22037,2 \\
\hline Balilla & 78642,2 & 17249,3 & 95891,5 & 3658,8 & 99550,3 \\
\hline Monte crisimo & 65465,2 & 14503,2 & 79968,4 & 3288,6 & 83257,0 \\
\hline Piano Porcheria & 18386,4 & 4207,8 & 22594,2 & 1487,7 & 24081,9 \\
\hline
\end{tabular}

des biomasses de 9,7, 39,7,60,5, 107,2 et $119,9 \mathrm{Mg} \mathrm{ha}^{-1}$ à $2,5,9,15$ et 19 ans.

La figure 4, qui regroupe l'ensemble de ces données, montre qu'il y a une excellente corrélation entre l'âge et les biomasses des stations françaises et l'âge et les biomasses de trois des stations de l'Etna. La station de Monte Crisimo présente, elle, des valeurs nettement différentes des trois autres sites de l'Etna, voisines des châtaigneraies de France. Ceci se retrouvera également au niveau de la productivité et résulte probablement d'une influence anthropique ancienne, la châtaigneraie actuelle occupant un ancien vignoble. À part le site de Monte Crisimo, la productivité de Castanea sativa est inférieure, de l'ordre de 30 à $40 \%$, dans le

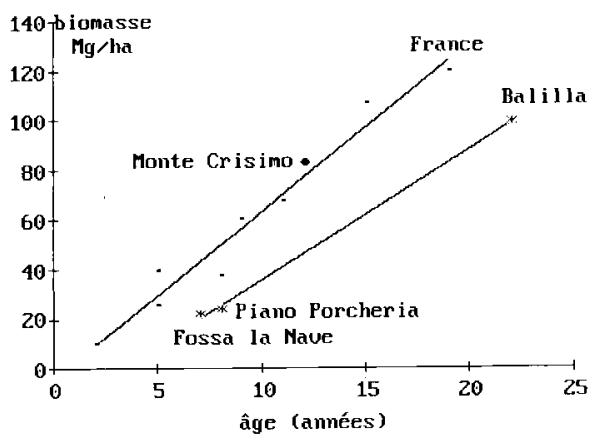

Fig 4. Comparaison de la relation âge / biomasse aérienne pour des peuplements de Castanea sativa de France (Berthier, 1984 ; Ranger, 1990) et de Sicile. massif de l'Etna par rapport aux peuplements plus septentrionaux.

Le tableau III indique également les parts respectives des différentes fractions. Les troncs représentent en moyenne $77 \%$ de la biomasse totale et $82 \%$ de la biomasse ligneuse épigée ; les branches, 17 et $18 \%$ alors que la part des feuilles est en moyenne de $6 \%$. Au niveau du tronc, l'écorce représente en moyenne $15 \%$ du poids total, avec des variations stationnelles se situant entre 13,4 et $16,4 \%$. Ranger et al (1990b) indiquent des pourcentages d'écorce allant de 10,1 à 15,8\% du poids du tronc, en relation inverse avec l'âge des peuplements.

Il faut également noter que les biomasses foliaires ont été déterminées en mai, donc avant la période de croissance maximale des feuilles. Une étude de la variation de la nappe foliaire au cours de la période de végétation faite dans les deux peuplements les plus jeunes (Fossa la Nave et Piano Porcheria) indique que la croissance se poursuit jusqu'en septembre.

Mais les peuplements étudiés, bien qu'exploités régulièrement toutes les 12 à 18 années, ne sont pas équiennes. Le silviculteur italien laissant toujours sur pied un certain nombre d'individus plus âgés, les « matricine ».

À partir des déterminations de l'âge des arbres abattus, de leur diamètre à $1,30 \mathrm{~m}$, de la démographie des quatre peuplements, il est possible d'estimer les biomasses pérennes respectives pour dif- 
Tableau IV. Répartition des biomasses individuelles par âge en poids $\mathrm{kg} \mathrm{ha}^{-1}$ dans les stations étudiées.

\begin{tabular}{|c|c|c|c|c|c|}
\hline \multirow[t]{2}{*}{ Station } & \multirow{2}{*}{$\begin{array}{c}\hat{A g e} \\
\text { (année) }\end{array}$} & \multirow{2}{*}{$\begin{array}{c}\text { Nombre } \\
\text { indiv } \\
\left(h a^{-1}\right)\end{array}$} & \multicolumn{3}{|c|}{ Poids (kg ha ${ }^{-1}$ ) } \\
\hline & & & Tronc & Branches & Bois \\
\hline Fossa la Nave & $\begin{array}{r}1 \\
7 \\
21 \\
35\end{array}$ & $\begin{array}{r}31 \\
1686 \\
164 \\
4\end{array}$ & $\begin{array}{r}12 \\
7578 \\
8692 \\
406\end{array}$ & $\begin{array}{r}3 \\
1729 \\
1921 \\
89\end{array}$ & $\begin{array}{r}15 \\
9307 \\
10613 \\
495\end{array}$ \\
\hline Balilla & $\begin{array}{r}1 \\
15 \\
30 \\
46 \\
60\end{array}$ & $\begin{array}{r}183 \\
852 \\
407 \\
70 \\
17\end{array}$ & $\begin{array}{r}29 \\
198843 \\
35965 \\
15859 \\
6946\end{array}$ & $\begin{array}{rr} & 7 \\
4330 \\
7993 \\
3429 \\
1490\end{array}$ & $\begin{array}{rr} & 36 \\
24173 \\
43958 \\
19288 \\
8436\end{array}$ \\
\hline Monte Crisimo & $\begin{array}{r}1 \\
10 \\
24 \\
38 \\
52\end{array}$ & $\begin{array}{r}1473 \\
3625 \\
345 \\
82 \\
4\end{array}$ & $\begin{array}{r}330 \\
32922 \\
17043 \\
13630 \\
1541\end{array}$ & $\begin{array}{r}79 \\
7364 \\
3769 \\
2961 \\
330\end{array}$ & $\begin{array}{r}409 \\
40286 \\
20812 \\
16591 \\
1871\end{array}$ \\
\hline Piano Porcheria & $=>21^{*}$ & $\begin{array}{r}1066 \\
4884 \\
12\end{array}$ & $\begin{array}{r}144 \\
16885 \\
1358\end{array}$ & $\begin{array}{r}34 \\
3876 \\
297\end{array}$ & $\begin{array}{r}178 \\
20761 \\
1655\end{array}$ \\
\hline
\end{tabular}

* Impossible d'estimer les individus âgés au-delà de 21 ans.

Tableau V. Évolution du poids des troncs et des branches $(\mathrm{kg})$ en fonction de l'âge des rejets.

\begin{tabular}{cccc}
\hline $\begin{array}{l}\text { Âge } \\
\text { (années) }\end{array}$ & $\begin{array}{c}\text { Poids de } \\
\text { l'arbre }\end{array}$ & $\begin{array}{c}\text { Origine } \\
\text { (station) }\end{array}$ & $\begin{array}{c}\text { Production } \\
\left(\mathrm{kg} \mathrm{an}^{-1}\right)\end{array}$ \\
\hline 7 & 5,49 & $\mathrm{FN}$ & 0,78 \\
7 & 4,25 & $\mathrm{PP}$ & 0,61 \\
10 & 11,11 & $\mathrm{MC}$ & 1,11 \\
15 & 28,32 & $\mathrm{Ba}$ & 1,89 \\
21 & 64,71 & $\mathrm{FN}$ & 3,08 \\
24 & $\mathrm{MC}$ & 2,51 \\
30 & 60,32 & $\mathrm{Ba}$ & 3,60 \\
35 & 108,00 & $\mathrm{FN}$ & 3,53 \\
38 & 123,75 & $\mathrm{MC}$ & 5,32 \\
46 & 202,33 & $\mathrm{Ba}$ & 5,99 \\
52 & 275,54 & $\mathrm{MC}$ & 8,35 \\
60 & 467,75 & $\mathrm{Ba}$ & 8,27 \\
\hline
\end{tabular}

$\mathrm{FN}=$ Fosa la Nave $; \mathrm{PP}=$ Piano Porcheria $; \mathrm{MC}=$ Monte Crisimo $; \mathrm{Ba}=$ Balilla

férentes classes d'âges (tableau IV) ou les biomasses individuelles des arbres en fonction de leur âge (tableau V). Ces dernières, exprimées en kilo par arbre, peuvent être définies par la régression:
Biomasse arbre $=0,0748 \times X^{2,156}$

avec $n=12 ; r=0,996 ; X$ : âge des arbres (années). 
Divisant ces biomasses d'arbre par leur âge respectif, on peut estimer la productivité moyenne annuelle d'un individu à 0,23 $\mathrm{kg} \mathrm{an}^{-1}$ par rejet d'un an ; 0,78-0,61 $\mathrm{kg} \mathrm{an}^{-1}$ par tige entre 1 et 7 ans (Fossa la Nave, Piano Porcheria) ; $1,11 \mathrm{~kg} \mathrm{an}^{-1}$ par tige entre 1 et 10 ans (Monte Crisimo) et 1,89

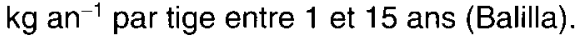

Les productivités calculées pour des arbres plus âgés (tableau V) sont plus aléatoires, car l'existence d'éventuels élagages peut entraîner une certaine sous-estimation.

Le même tableau permet également d'estimer un âge moyen pour chaque station à partir des classes d'âge et du nombre d'individus par classe. On obtient les âges moyens respectifs suivants : $7 / 8$ ans à Piano Porcheria, 8 ans à Fossa la Nave, 12 ans à Monte Crisimo, 22 ans à Balilla.

Le tableau VI résume la valeur des différentes biomasses déterminées à 2 ou 3 ans d'intervalle dans les stations de Fossa la Nave, Balilla et Monte Crisimo suite à une nouvelle mesure des diamètres des différentes tiges. Pour la station de Piano Porcheria, la seconde valeur de biomasse n'est pas le résultat des mensurations directes, mais d'un calcul effectué selon l'approche indiquée dans la partie méthodes d'étude.

À partir de ces valeurs on obtient pour chaque peuplement une production aérienne pérenne stationnelles de $2435 \mathrm{~kg} \mathrm{ha}^{-1}$ $a^{-1}$ à Fossa la Nave, 3257 à Balilla, 5359 à Monte Crisimo, 4381 à Piano Porcheria.
Ces résultats confirment la nette différence dans la productivité des quatre peuplements, qui est en relation directe avec l'altitude, donc avec les conditions climatiques stationnelles déjà évoquées et avec l'âge, donc la maturité des parcelles étudiées.

Les stations avoisinant $1500 \mathrm{~m}$, avec une exposition sud, ont une productivité moyenne annuelle faible, avec respectivement 2,4 et $3,3 \mathrm{Mg} \mathrm{ha}^{-1} \mathrm{an}^{-1}$ au bout de 7 et de 22 années. Celles plus basses, du versant est du volcan, présentent, elles, des productivités moyennes annuelles de 4,4 et de $5,4 \mathrm{Mg} \mathrm{ha}^{-1} \mathrm{an}^{-1}$, aux âges respectifs de 7 et de 12 ans. Ceci résulte à la fois des températures plus élevées, durant une période beaucoup plus longue aux altitudes plus basses, mais surtout d'une pluviométrie également plus importante. Cette dernière a été de $667 \mathrm{~mm}$ à Fossa la Nave et de $964 \mathrm{~mm}$ à Piano Porcheria durant l'année 1992 (Leonardi et al, 1993).

Ces valeurs sont dans la fourchette signalée par Cole et Rapp (1981). Concernant plus particulièrement Castanea sativa, Ranger et al $(1990 \mathrm{a}, \mathrm{b})$ indiquent des productions annuelles moyennes dépassant les $6 \mathrm{Mg} \mathrm{ha}^{-1} \mathrm{an}^{-1}$ à 19 et à 23 ans en France. Bedeneau $(1988,1994)$ cite des incréments annuels moyens de $4,3 \mathrm{Mg} \mathrm{ha}^{-1}$ à 9 ans et $3,0 \mathrm{Mg} \mathrm{ha}^{-1}$ à 12 ans, avec des valeurs maximales de $12 \mathrm{Mg} \mathrm{ha}^{-1} \mathrm{an}^{-1}$ à 9 ans et $8 \mathrm{Mg} \mathrm{ha}^{-1} \mathrm{an}^{-1}$ à 12 ans. Le même auteur signale également l'interférence de la forte mortalité avant l'âge de 15 ans sur

Tableau VI. Biomasses $\left(\mathrm{kg} \mathrm{ha}^{-1}\right)$ déterminées à 2 ou 3 ans d'intervalle et productivité aérienne pérenne $\left(\mathrm{kg} \mathrm{ha}^{-1} \mathrm{an}^{-1}\right)$ des quatre stations.

\begin{tabular}{lccccc}
\hline & $\begin{array}{c}\text { Biomasse } \\
1990\end{array}$ & $\begin{array}{c}\text { Biomasse } \\
1992 / 93^{*}\end{array}$ & $\begin{array}{c}\text { Production } \\
\text { moyenne } \\
\text { annuelle }\end{array}$ & $\begin{array}{c}\text { Production } \\
\text { tronc }\end{array}$ & $\begin{array}{c}\text { Production } \\
\text { branches }\end{array}$ \\
\hline Fosse la Nave & 20430 & $27735^{\star}$ & 2435 & 1991 & 444 \\
Balilla & 95891 & 102405 & 3257 & 2689 & 568 \\
Monte crisimo & 79968 & 90759 & 5395 & 4417 & 978 \\
Piano Porcheria & 22466 & $31229^{\star *}$ & 4381 & 3592 & 789 \\
\hline
\end{tabular}

" Fossa la Nave 3 années ; ${ }^{\star \star}$ valeur calculée pour tenir compte de la mortalité des jeunes rejets 
Tableau VII. Production de biomasse pérenne $\left(\mathrm{Mg} \mathrm{ha}^{-1}\right)$ pour un indice foliaire de 1.

\begin{tabular}{lcc}
\hline & Stations d'altitude & Stations plus basses \\
\hline Peuplement âgé & 0,6 (Balilla) & 1,1 (Monte Crisimo) \\
Peuplement jeune & 1,6 (Fossa la Nave) & 2,2 (Piano Porcheria) \\
\hline
\end{tabular}

la productivité des taillis de Castanea sativa Mill. Berthier (1984) détermine une production primaire nette de 6,3 et de 6,7 $\mathrm{Mg} \mathrm{ha}^{-1} \mathrm{an}^{-1}$ pour des taillis de Castanea sativa âgés respectivement de 8 et 11 ans, dans le sud-est de la France.

Ces différences de productivité se retrouvent et se répercutent également au niveau de l'indice foliaire des quatre stations. Dans les taillis jeunes, celui-ci est de 1,5 (Fossa la Nave) et de 2,0 (Piano Porcheria). Il atteint respectivement 5,0 et 5,4 à Monte Crisimo et à Balilla. Mais, comme déjà signalé pour la biomasse foliaire, ces valeurs sont sous-estimées, car les feuilles continuent à croître longtemps après la mesure de leur surface, effectuée au mois de mai.

II semble donc que, pour la nappe foliaire, on a également affaire à deux mécanismes qui se superposent : l'âge d'un côté, l'indice foliaire augmentant avec la taille de plus en plus grande des arbres et à nouveau les facteurs climatiques locaux de l'autre côté.

Si l'on ramène ces productions respectives à un même indice foliaire, on remarque que, pour un indice foliaire de 1 , les peuplements plus jeunes produisent davantage de biomasse que les peuplements âgés. De même, les peuplements implantés à faible altitude, produisent à âge égal, plus que les peuplements d'altitude (tableau VII).

Nous retrouvons encore l'important rôle des facteurs climatiques qui se superposent aux effets de l'âge. Ceci résulte essentiellement du fait que les peuplements âgés investissent davantage dans les énergies de maintenance que dans la production apparente.

\section{Restitution au sol de matière organique par l'intermédiaire de la litière}

Au cours de 3 années consécutives on a déterminé, pour chacun des quatre sites, les retombées de litière suivantes en $\mathrm{kg} \mathrm{ha}^{-1}$ $\mathrm{an}^{-1}$ (tableau VIII).

On retrouve encore la nette difference entre les deux taillis âgés et les deux taillis coupés récemment. Ranger et al (1990b) aboutissent aux mêmes résultats avec des retombées de litière de l'ordre de $2,5 \mathrm{Mg}$ $\mathrm{ha}^{-1} \mathrm{an}^{-1}, 2$ ans après la coupe à blanc et $5,0 \mathrm{Mg} \mathrm{ha}^{-1} \mathrm{an}^{-1}$ pour des peuplements âgés de 10 à 20 ans.

Les feuilles représentent $51 \%$ de la litière dans les stations de Fossa la Nave, Balilla et Monte Crisimo et $72 \%$ à Piano Porcheria. Ceci provient essentiellement de la très faible production d'organes reproducteurs et de fruits dans cette dernière station.

Tableau VIII. Retombée de litière dans les quatre stations étudiées.

\begin{tabular}{lcc}
\hline & $\begin{array}{c}\text { Litière totale } \\
\left(\mathrm{kg} \mathrm{ha} \mathrm{an}^{-1}\right)\end{array}$ & $\begin{array}{c}\text { Litière feuilles } \\
\left(\mathrm{kg} \mathrm{ha} \mathrm{an}^{-1}\right)\end{array}$ \\
\hline Fossa la Nave & 2640 & 1353 \\
Batilla & 5822 & 2984 \\
Monte Crisimo & 5093 & 2664 \\
Piano Porcheria & 1709 & 1230 \\
\hline
\end{tabular}


Notons également que, par rapport à la biomasse foliaire, la fraction « feuilles » de la litière indique, dans les quatre cas, une perte de $18 \%$ lors de la sénescence de ces organes.

La fraction bois, constituée de brindilles et de petits rameaux, est peu importante dans les litières des jeunes taillis (66 et 26 $\mathrm{kg} \mathrm{ha}^{-1} \mathrm{an}^{-1}$ ) comparée aux deux stations les plus âgées (741 et $1428 \mathrm{~kg} \mathrm{ha}^{-1} \mathrm{an}^{-1}$ ). En réalité la nécromasse ligneuse est plus élevée à Piano Porcheria, mais les 713 jeunes tiges qui ont disparu en deux ans ne sont pas prises en compte par le dispositif de récolte des litières. Ranger et al (1990b) signalent également le rôle non négligeable du bois dans la litière des châtaigneraies, qui augmente par ailleurs parallèllement à l'âge des taillis.

\section{Accumulation de litière au sol}

Le tableau IX résume l'ensemble des déterminations pondérales de la litière immobilisée à la surface du sol, avec l'erreur standard entre les dix prélèvements effectués dans chaque station, à la fois globalement et par fraction de litière. Ces valeurs sont exprimées en matière organique après calcination.

On note que le stock de litière au sol est relativement uniforme, variant entre 7,4 et $7,9 \mathrm{Mg} \mathrm{ha}^{-1}$, sans relation apparente avec l'âge réel des différents taillis. Mais on remarque en revanche des différences sensibles entre les fractions identifiées. Ainsi la litière ligneuse est trois fois plus importante dans les peuplements âgés par rapports aux stations récemment coupées. $\mathrm{Ceci}$ semble indiquer que l'effet des coupes, entreprises 7 ans avant les observations à Fossa la Nave et à Piano Porcheria, ne s'est pas traduit par une accélération du processus de décomposition de la litière. Bien plus, les deux stations les plus récemment coupées présentent les stocks de feuilles les plus élevés, malgré des retombées plus faibles de cette fraction de la litière au cours des dernières années. Contrairement aux effets d'augmentation et d'accélération de la décomposition, induits par les coupes forestières en climat tempéré ou froid, on semble assister à un freinage de la décomposition en région méditerranéenne. Parmi les hypothèses que l'on peut avancer pour expliquer ce fait, on peut citer : i) les fortes températures estivales qui empêchent toute activité microbienne et ii) la dessiccation de la nécromasse végétale se prolongeant longtemps en automne et en hiver et inhibant également l'activité des décomposeurs.

Signalons que Ranger et al (1990b) indiquent une masse holorganique de $15 \mathrm{Mg}$ ha $^{-1}$ à 15 ans, ce qui est plus du double du stock déterminé dans le taillis de Monte Crisimo, âgé de 12 ans.

Cette faible accumulation de litière à la surface du sol témoigne néanmoins d'une très bonne décomposition relative dans les conditions spécifiques de l'Etna, grâce encore, à une conjonction entre les effets de la température et des précipitations.

\section{Décomposition de la litière}

À partir de l'estimation des retombées annuelles de litière et des stocks de litière immobilisés à la surface du sol il est possible

Tableau IX. Stock de litière au sol $\left(\mathrm{kg} \mathrm{ha}^{-1}\right)$ dans les quatre taillis, déduction faite des cendres.

\begin{tabular}{lcrrr}
\hline & Feuilles & $\begin{array}{c}\text { Organes } \\
\text { reproducteurs }\end{array}$ & Bois & Total \\
\hline Fossa la Nave & $5497 \pm 433,8$ & $1910 \pm 221,2$ & $332 \pm 103,6$ & 7739 \\
Balilla & $5230 \pm 652,9$ & $1205 \pm 183,4$ & $956 \pm 234,0$ & 7391 \\
Monte Crisimo & $4461 \pm 257,3$ & $2525 \pm 363,5$ & $880 \pm 110,9$ & 7876 \\
Piano Porcheria & $7192 \pm 502,6$ & $425 \pm 132,8$ & $100 \pm 30,3$ & 7717 \\
\hline
\end{tabular}


Tableau X. Taux de décomposition et estimations des différents temps de décomposition calculés selon Jenny et al (1949).

\begin{tabular}{lcc}
\hline & Balilla & Monte Crisimo \\
\hline Litière totale & & \\
Coefficient de Jenny (\%) & 40,4 & 39,3 \\
Temps de demi-décomposition (années) & 1,3 & 1,4 \\
Temps de 95\% de décomposition (années) & 5,8 & 6,0 \\
& & \\
Fraction feuilles & 29,4 & 37,4 \\
Coefficient de Jenny (\%) & 2,0 & 1,5 \\
Temps de demi-décomposition & 8,6 & 6,4 \\
Temps de 95\% de décomposition & & \\
\hline
\end{tabular}

de calculer globalement un certain nombre d'indices de décomposition en condition naturelle, proposés par Jenny et al (1949) ou Olson (1963).

Si ces indices, qui supposent que les taillis étudiés sont en équilibre, peuvent aisément s'appliquer aux deux stations de Balilla et de Monte Crisimo, leur utilisation pour les peuplements jeunes, comme ceux des deux autres stations étudiées dans le massif de l'Etna, est plus aléatoire.

Nous avons calculé pour les deux taillis les plus âgés les coefficients de Jenny et al (1949) et les temps respectifs de demi-décomposition et de $95 \%$ de décomposition de la litière, pour la litière totale et pour la fraction des feuilles prise seule (tableau $X$ ).

$S i$ les valeurs sont pratiquement similaires entre les deux stations en ce qui concerne les résultats des processus biologiques de décomposition de la litière globale, elles divergent au niveau de la fraction feuilles.

Mais nous avons également estimé la décomposition, du moins celle des feuilles par une seconde approche. À cet effet, différents échantillons de feuilles de Castanea sativa ont été mis à décomposer dans des litter-bags durant une période de deux ans dans les quatre châtaigneraies de l'Etna. Les résultats, toutes stations regroupées (fig 5), indiquent en premier lieu une similitude du processus quantitatif de la décom- position, complètement indépendant de sa localisation. Seuls les facteurs macroclimatiques semblent jouer un rôle important dans ce processus, en l'absence de toute différence édaphique d'une station à l'autre.

La figure 5 représente également l'allure générale de la décomposition des feuilles de Castanea sativa durant ces deux années. Elle correspond à une régression de type puissance répondant à l'équation :

$$
Y=169,20 X^{-0,17}
$$

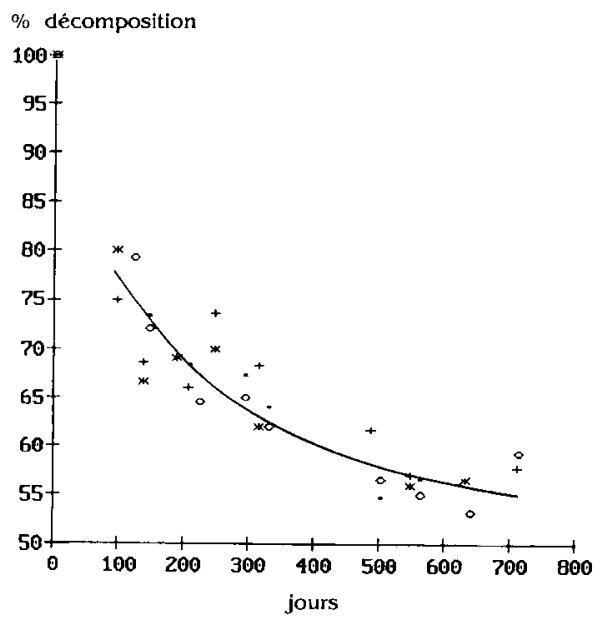

Fig 5. Décomposition des feuilles de Castanea sativa en "litter-bag" durant deux années consécutives dans les quatre stations de l'Etna. 
avec $Y, \%$ décomposition ; $X$, jours ; $n=32 ; r=0,847$.

Cette première régression, intégrant les deux années d'observation, peut se subdiviser elle-même en deux régressions linéaires, représentative chacune d'une année (fig 6).

La décomposition est plus importante la première année, représentée par l'équation de régression linéaire :

$$
Y(\%)=92,5-0,1 X \text { (jours) }(r=0,867)
$$

Elle diminue sensiblement la deuxième année, comme le montre la constante « $b$ " très faible dans l'équation :

$$
Y(\%)=60,95-0,01 \times(\text { jours })
$$

Mais cette faible décomposition introduit une plus grande marge d'incertitude dans l'expression linéaire que dans les régressions puissances ou logarithmiques.

À partir de cette équation, on peut déterminer un taux de décomposition de $35 \%$ à la fin de la première année. On note une excellente corrélation de cette valeur par rapport à l'approche de Jenny et al (1949).

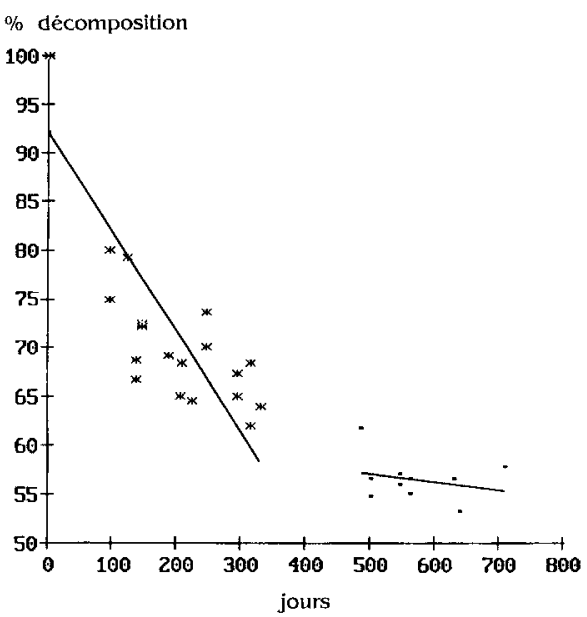

Fig 6. Décomposition annuelle des feuilles de Castanea sativa durant 2 années successives.
La méthode de Jenny et al (1949) indique que la moitié de la litière est décomposée en 1,3 et 1,4 ans, alors que la valeur obtenue expérimentalement est de $35 \%$ de décomposition lors de la première année. Les deux approches se recouvrent en valeur et en temps. Des différences dans les processus de décomposition entre litterbag et méthode de Jenny et al (1949) ont été signalées par Rapp (1971). II semble que les conditions microclimatiques créées à l'intérieur des sachets de litière (meilleur rétention de l'eau lors des périodes de sécheresse) favorisent la décomposition des feuilles en litter-bag, et ce malgré une diminution probable de l'activité faunistique, suite au maillage des sacs.

\section{Comparaison des quatre sites}

Comme indiqué sur le tableau I, les sols sont relativement identiques. Les seules différences notables sont liées à leur localisation. Les sols des deux stations de basse altitude sont en général plus pauvres en bioéléments totaux, exception faite du magnésium. De même leur capacité d'échange, les cations échangeables $(\mathrm{K}, \mathrm{Ca}$ et $\mathrm{Mg}$ ) et les réserves organiques ( $\mathrm{C}$ et $\mathrm{N}$ ) sont un peu inférieures à ce qui est observé pour les sols des peuplements d'altitude.

Les deux variables essentielles sont donc représentées par les facteurs climatiques (altitude, exposition) et l'âge des peuplements.

Nous avons regroupé sur la figure 7 l'ensemble des valeurs concernant les différents compartiments et flux de la matière organique, selon l'altitude des stations et l'âge moyen des arbres.

À partir de ces données, on peut calculer un certain nombre de rapports et de relations entre les quatre peuplements en fonction de ces deux paramètres.

Ainsi la production annuelle de biomasse pérenne est selon l'altitude de 4,4 et $2,4 \mathrm{Mg}$ ha ${ }^{-1} \mathrm{an}^{-1}$ pour les deux peuplements les plus jeunes et de 5,4 et $3,3 \mathrm{Mg} \mathrm{ha}^{-1} \mathrm{an}^{-1}$ pour les deux stations plus âgées de Monte 


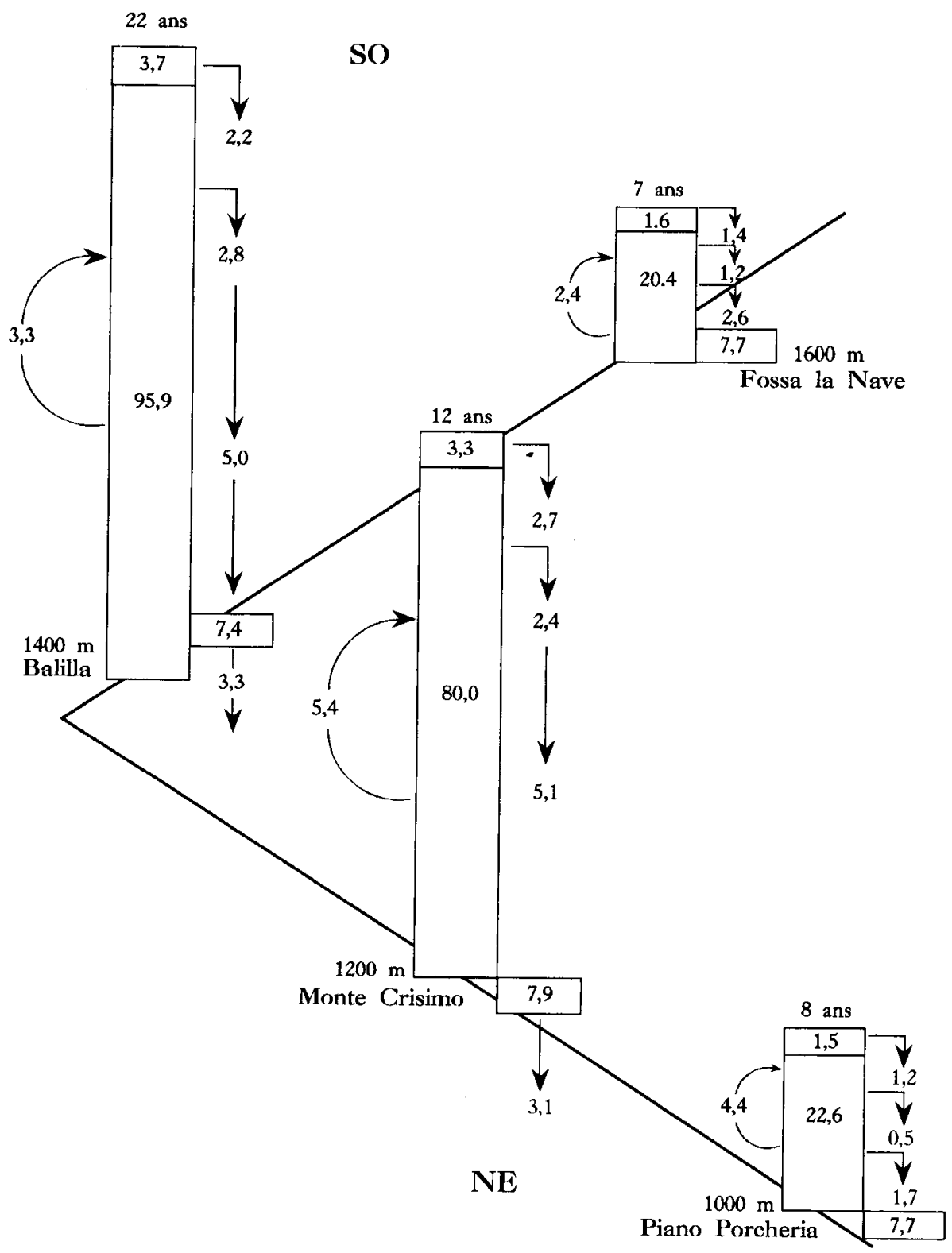

Fig 7. Représentation synthétique et quantitative des différents compartiments et flux de la matière organique dans les quatre stations de l'Etna. Les compartiments : bois, feuilles, litière au sol, sont exprimés en $\mathrm{Mg} \mathrm{ha}^{-1}$. Les flux (matérialisés par des flèches) : litière feuilles, autre litière, litière totale, décomposition de la litière, incrément annuel du bois, sont exprimés en $\mathrm{Mg} \mathrm{ha}^{-1} \mathrm{an}^{-1}$. 
Tableau XI. Relations entre la productivité pérenne en fonction de la biomasse foliaire et l'âge ou la localisation altitudinale des quatre peuplements.

\begin{tabular}{lcccc}
\hline & $\begin{array}{c}\text { Biomasse } \\
\text { feuilles } \\
\left(\mathrm{Mg} \mathrm{ha}^{-1}\right)\end{array}$ & $\begin{array}{c}\text { Production } \\
\text { pérenne } \\
\left(\mathrm{Mg} \mathrm{ha}^{-1} \mathrm{an} \mathrm{n}^{-1}\right)\end{array}$ & $\begin{array}{c}\text { Production pérenne } \\
\text { pour }\end{array}$ & $\begin{array}{c}\text { Rapport : } \\
\text { station jeune/ } \\
\text { station âgée }\end{array}$ \\
\hline Fossa la Nave & 1,6 & 2,4 & 1,5 & \\
Balilla & 3,7 & 3,3 & 0,9 & 1,7 \\
Piano Porcheria & 1,5 & 4,4 & 2,9 & 1,8 \\
Monte Crisimo & 3,3 & 5,4 & 1,6 & 1,8 \\
\hline
\end{tabular}

Crisimo et de Balilla. Cela confirme que la production diminue bien avec l'altitude de l'implantation des taillis de Castanea sativa. Mais l'âge du peuplement joue également un rôle. Ceci est confirmé si l'on exprime, pour pouvoir comparer les parcelles, la production moyenne de matériel pérenne par rapport à une quantité donnée $(1 \mathrm{Mg})$ de matériel foliaire (tableau XI). L'on retrouve les différences altitudinales avec respectivement 1,5 et $0,9 \mathrm{Mg} \mathrm{ha}^{-1}$ de matériel pérenne produit en altitude et 2,9 et $1,6 \mathrm{Mg} \mathrm{ha}^{-1}$ entre 1000 et $1200 \mathrm{~m}$. Mais les rapports : station jeune/station âgée sont très proches : 1,7 et 1,8 .

Le rôle de l'âge des arbres apparaît également au niveau de la production primaire nette (PPN = incrément pérenne + retombée de litière). Le tableau XII indique que l'incrément de matériel pérenne dépend essentiellement des facteurs climatiques, avec un rapport moyen : station basse / station d'altitude (B/A) de 1,73 contre un rapport moyen : station âgée / station jeune $(A / J)$ de 1,30 .
À l'opposé, en ce qui concerne la production primaire nette, par suite du rôle prépondérant de la biomasse foliaire, qui augmente avec l'âge, c'est ce dernier facteur qui joue le rôle primordial : $\mathrm{A} / \mathrm{J}$ moyen $=1,69$ et $\mathrm{B} / \mathrm{A}$ moyen $=1,24$.

L'altitude ne semble pas influencer le recyclage de la matière organique, qui se fait également plutôt une fonction de l'âge. Ainsi les arbres jeunes produisent davantage de litière, comparée à la biomasse renne, que les individus âgés, surtout sil'on prend en compte la sous-évaluation à Piano Porcheria, suite à l'importante mortalité constatée dans cette station.

Quant aux processus de décomposition, on ne note pas de différence entre les deux stations âgées en fonction de l'altitude. Les taux de décomposition, calculés selon Jenny et al (1949) sont pratiquement identiques, de l'ordre de $40 \%$, ce qui représente une disparition annuelle de 3,1 à $3,3 \mathrm{Mg}$ $\mathrm{ha}^{-1}$ de litière.

Les processus de décomposition n'ont pas été étudiés dans les deux stations ex-

Tableau XII. Production annuelle pérenne et production primaire nette en fonction de l'âge et de la localisation altitudinale des quatre peuplements.

\begin{tabular}{lcccccc}
\hline & \multicolumn{3}{c}{ Production annuelle pérenne } & \multicolumn{3}{c}{ Production primaire nette } \\
\cline { 2 - 7 } \multicolumn{1}{c}{ Taillis : } & Jeunes $(\mathrm{J})$ & $\hat{\text { Agés }}(\mathrm{A})$ & $\mathrm{A} / \mathrm{J}$ & Jeunes $(\mathrm{J})$ & $\hat{\text { Agés }}(\mathrm{A})$ & $\mathrm{A} / \mathrm{J}$ \\
\hline Stations d'altitude (A) & 2,4 & 3,3 & 1,37 & 5,0 & 8,3 & 1,66 \\
Stations basses (B) & 4,4 & 5,4 & 1,23 & 6,1 & 10,5 & 1,72 \\
B / A & 1,83 & 1,64 & & 1,22 & 1,26 & \\
& & & & & &
\end{tabular}


Tableau XIII. Comparaison des différents paramètres du cycle de la matière organique, exprimés en pourcent d'une biomasse aérienne commune : $100 \mathrm{~kg} \mathrm{ha}^{-1}$ de matériel végétal.

\begin{tabular}{|c|c|c|c|c|}
\hline & \multicolumn{2}{|c|}{ Stations basses } & \multicolumn{2}{|c|}{ Stations d'altitude } \\
\hline & Jeunes & Âgées & Jeunes & Âgées \\
\hline Biomasse épigée & 100 & 100 & 100 & 100 \\
\hline \multicolumn{5}{|l|}{ Retombée litière } \\
\hline Feuilles (\%) & 5,3 & 3,4 & 6,9 & 3,1 \\
\hline Total $(\%)$ & 7,5 & 6,4 & 12,7 & 6,0 \\
\hline \multicolumn{5}{|l|}{ Litière au sol } \\
\hline Feuilles (\%) & 31,8 & 5,6 & 27,0 & 5,4 \\
\hline Total (\%) & 34,1 & 9,9 & 37,7 & 7,7 \\
\hline Décomposition & & 3,9 & & 3,4 \\
\hline \multicolumn{5}{|l|}{ Production } \\
\hline Incrément (\%) & 19,5 & 6,7 & 11,8 & 3,4 \\
\hline Feuilles (\%) & 5,3 & 3,4 & 6,9 & 3,1 \\
\hline Total $(\%)$ & 24,8 & 10,1 & 18,7 & 6,5 \\
\hline
\end{tabular}

ploitées récemment, car l'on ne peut pas considérér qu'elles soient parvenues à un stade d'équilibre, après la perturbation subie lors de la coupe à blanc, malgré un stock global de litière au sol semblable à celui déterminé dans les deux peuplements âgés.

Ces différences inter-stationnelles sont encore mieux perceptibles si l'on exprime les différentes quantités de la figure 4 en pourcentage de la biomasse aérienne pérenne pour toutes les quatre stations (tableau XIII).

On retrouve l'important recyclage de matière organique sous forme de litière dans les stations les plus jeunes, jumelée à une forte productivité primaire. Les deux peuplements âgés montrent peu de différences en ce qui concerne les flux cataboliques : chute de litière, accumulation et décomposition de la litière. À l'opposé, l'influence climatique se traduit par une production plus élevée dans la station de faible altitude (1 $200 \mathrm{~m})$ par rapport à la châtaigneraie située à $1400 \mathrm{~m}$.

\section{CONCLUSIONS}

La comparaison des différents compartiments et flux de matière organique dans quatre taillis de Castanea sativa Mill, d'âge différent et implantés sur un gradient altitudinal dans le massif de l'Etna (Sicile) met en évidence quatre conclusions.

i) Les différentes étapes du cycle sont influencées par l'un ou l'autre de ces paramètres (âge, climat) ou les deux ensemble.

ii) La productivité pérenne est en relation avec l'altitude, donc avec les facteurs climatiques, alors que la production primaire nette est d'avantage fonction de l'âge des taillis. Ce dernier fait est la conséquence d'une plus grande masse foliaire dans les peuplements matures.

iii) La décomposition, calculée selon Jenny et al (1949), semble totalement indépendante 
des conditions climatiques stationnelles. C'est le climat méditerranéen, caractérisé par une période estivale prolongée, chaude et sèche, qui imprime sa marque sur ce processus, occultant les différences microclimatiques.

iv) Du point de vue cinétique, c'est encore l'âge des peuplements qui semble être primordial. Mais ce sont les taillis les plus jeunes qui présentent, proportionnellement, un plus grand recyclage de matériel organique à travers le système, comparés aux stations plus âgées.

\section{REMERCIEMENTS}

Les auteurs tiennent à remercier les docteurs A Drago et G Scrimali, de l'Ispettorato Ripartimentale Agricoltura e Foreste de Catania, qui ont mis à leur disposition les parcelles d'étude et toute l'aide nécessaire aux travaux de terrain. Cette recherche a été financée par l'UE, contrat STEP/PL 900257 « Biological criteria for sustainable development in natural degenerate forests of Mediterranean Europe ».

\section{RÉFÉRENCES}

Asciuto G, Crescimanno M, Raimondo A, (1988) II castagno in Sicilia. Cellulosa e Carta 39, 5, 4-11

Baskerville GL (1965) Estimation of dry weight of tree components and total standing in conifer stands. Ecology 46, 867-869

Berthier B (1984) Fonctionnement d'un écosystème forestier : croissance, biomasse et productivité du compartiment ligneux épigé des taillis de châtaigniers Castanea sativa Mill du sud-est de la France. Thèse, univ de Grenoble, $62 \mathrm{p}$

Bedeneau M (1988) Croissance du taillis de châtaignier en France : premiers résultats. Ann Sci For 45, 3, 265-274

Bedeneau M (1994) Croissance des taillis de châtaignier en France : utilisation des données de l'Inventaire forestier national. Ann Sci For 51, 2, 183-194

Bocock KL, Gilbert OJW (1957) The disappearance of leaf litter under different woodland conditions. Plant Soil $9,179-185$

Boggia L (1988). Conclusioni sulla castanicoltura nazionale. Cellulosa Carta 39, 4-10

Bouchon J, Nys C, Ranger J (1985) Cubage, biomasse et minéralomasse : comparaison de trois taillis simples des Ardennes primaires. Acta Oecol, Oecol Plant 6, 53-72
Burges A (1956) The release of cations during the decomposition of forest litter. $\mathrm{CR} \mathrm{VI}{ }^{\circ} \mathrm{C} / \mathrm{it}$ Sci Soil Paris, B 1/2, 741-745

Chatelus $S$ (1987). Contribution à l'analyse du cycle biologique des éléments minéraux dans un écosystème forestier : cas particulier du taillis de châtaignier (Castanea sativa Mill). Thèse, univ de Limoges, $151 \mathrm{p}$

Cole DW, Rapp M (1981) Elemental cycling in forest ecosystem. In : Dynamic Properties of Forest ECOsystems (DE Reichle, ed), Cambridge Univ Press, Cambridge, 341-409

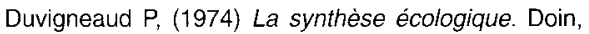
Paris, $296 p$

Ford ED, Newbould PJ (1970) Stand structure and dry weight production through the sweet chestnut (Castanea sativa Mill) coppice cycle. J Ecol 58, 275-296

Hanchi A (1994) Cycle de l'eau et des éléments biogènes dans un bassin versant forestier : cas d'une hêtraie au mont Lozère. Thèse, univ de Dijon, $232 p$

Jenny H, Gessel S, Bingham FT (1949) Comparative study of decomposition rates of organic matter in temperate and tropical regions. Soil Sci 2, 419-432

La Marca O (1984) Ricerche sulla biomassa dei cedui di castagno (Castanea sativa Mill) della Valle delI'Irno (AV e SA). Ricerche Sperimentali di Dendrometria e di Auxometria, Cappini, Florence, 8, 63-79

Lemée $G$ (1974) La production primaire de la forêt. In : Écologie forestière (P Pesson, ed), Gauthier-Villars, Paris, 135-153

Leonardi S, Rapp M, Failla M, Guarnaccia D (1993) Interception of rainfall, input and leaching of nutrients within two Castanea sativa stands at the Etna volcano. Oecologia Montana 2, 7-12

Olson J S (1963) Energy storage and the balance of producers and decomposers in ecological systems. Ecology 44, 2, 322-331

Poli E, Maugeri G, Ronsisvalle GA (1981) Note illustrative della carta della vegetazione dell'Etna. CNR, $A Q / 1 / 131$, Roma

Ranger J, Nys C, Bouchon J (1990a) Les relations entre la fertilité du sol, la production et l'utilisation d'éléments nutritifs dans les taillis de châtaigniers. Acta Oecol 11, 487-501

Ranger J, Felix C, Bouchon J, Nys C, Ravart M (1990b) Dynamique d'incorporation du carbone et des éléments nutritifs dans un taillis simple de châtaignier (Castanea sativa Miller). Ann Sci For 47, 413-433

Rapp M (1971) Cycle de la matière organique et des éléments minéraux dans quelques écosystèmes méditerranéens. In : Écologie du sol. Recherche coopérative sur programme, CNRS, Paris, 40, 19188

Ronsisvalle GA, Signorello P (1979) Contributo allo studio fitosociologico dei castagneti dell'Etna. Boll ACC Gioenia Sc Nat Catania 4, 29-41

Scuderi S (1825) Trattato dei boschi dell'Etna. Atti ACC Gioenia Sc Nat Catania. 